\title{
A Review on Substrate Integrated Waveguide and its Microstrip Interconnect
}

\author{
Hemendra Kumar ${ }^{1}$, Ruchira Jadhav ${ }^{2}$, Sulabha Ranade ${ }^{3}$ \\ ${ }^{1,2}$ (EXTC Department, K.J. Somaiya College of Engineering, University of Mumbai, India) \\ ${ }^{3}$ (SAMEER, IIT Campus, Powai, India)
}

\begin{abstract}
Substrate Integrated Waveguides (SIW) are used for transmission of Electromagnetic waves. They are planar structures belonging to the family of Substrate Integrated Circuits. Because of their planar nature, they can be fabricated on planar circuits like Printed Circuit Boards (PCB) and can be integrated with other planar transmission lines like microstrips. They retain the low loss property of their conventional metallic waveguides and are widely used as interconnection in high speed circuits, filters, directional couplers, antennas. This paper is a comprehensive review of Substrate Integrated Waveguide and its integration with Microstrip line. In this paper, design techniques for SIW and its microstrip interconnect are presented. HFSS is used for simulation results. The objective of this paper is to provide broad perspective of SIW Technology.
\end{abstract}

Keywords: Microstrip Transition, Reflection Parameter, Substrate Integrated Waveguide, Vias.

\section{Introduction}

The Transmission lines like two-wire cables and coaxial cables are traditionally used for transmission of Electromagnetic energy. These transmission lines result in lot of radiation loss, copper loss, skin effect and dielectric loss. Moreover they are non-planar in nature, so their integration with planar circuit and its components is complex. Metallic waveguides are better wave guiding options then compared to traditional transmission lines. But, they are bulky and non-planar in nature. Substrate Integrated Waveguide (SIW) acts as alternative option to metallic waveguides. SIW are planar structures fabricated using two periodic rows of metallic vias (holes) or slots connecting top and bottom metallic ground planes of dielectric substrate as shown in Fig.1 below. Using SIW Technology a non-planar metallic waveguide can be modeled into a Substrate Integrated Waveguide, which will be planar in nature and can be fabricated on, as well as integrated to, planar circuits with ease. In [1], some active and passive SIW components are discussed. Like microstrips and coplanar lines SIW components are compact, light, cost effective and easy to fabricate.

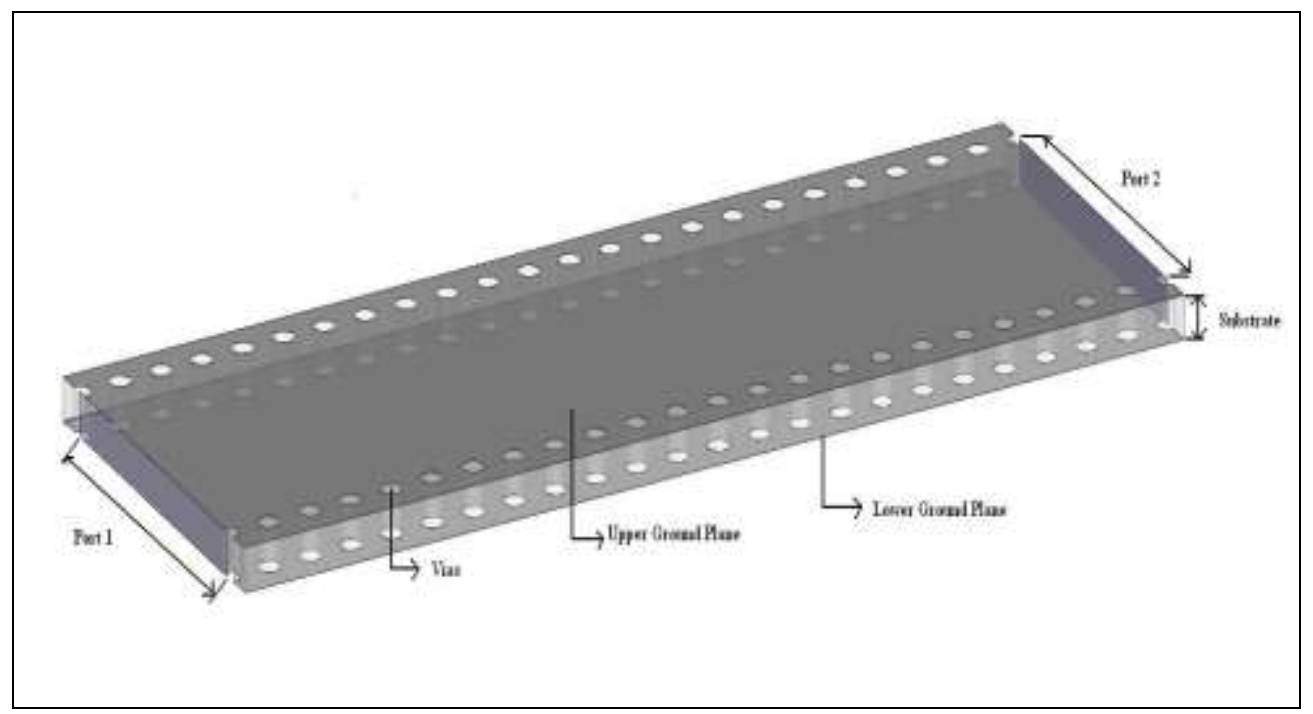

Fig. 1 Substrate Integrated Waveguide

\section{Design Technique For SIW}

We consider here Rectangular SIW to understand design technique of SIW. For Rectangular SIW the width and height are important consideration because the cutoff frequency of it depends on them. For a rectangular waveguide TE10 mode is dominant mode. Below Fig. 2 shows SIW model and the parameters to be considered. 


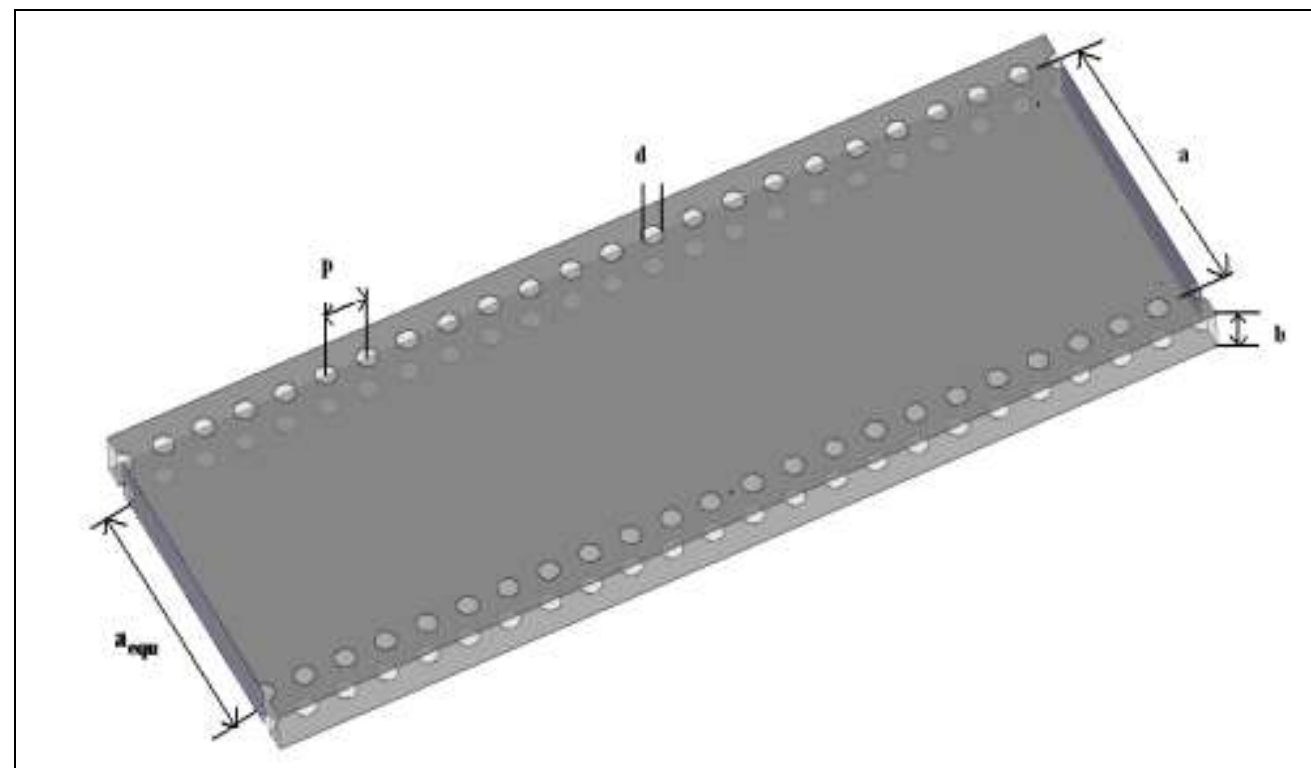

Fig. 2 Parameters of SIW

The above shown parameters play important role in designing SIW. ' $\mathbf{p}$ ' is the period between the vias, ' $\mathbf{d}$ ' is diameter of vias, ' $\mathbf{b}$ ' is the height of substrate, ' $\mathbf{a}$ ' is center to center distance between vias of both the rows, substrate dielectric constant ' $\square$ ' ' and ' $\mathbf{a}_{\text {equ }}$ ' is the width of dielectric filled metallic waveguide (equivalent to its vias SIW counterpart) discussed in [2].

In [3] conventional air filled waveguide to its equivalent dielectric filled waveguide mapping is discussed. If ' $\mathbf{a}_{\text {con }}$ ' is the width of conventional wave guide and ' $\square_{\text {con' }}$ ' is 1 (air) than its equivalent dielectric filled waveguide width is formulated as from [3]:

$$
\mathrm{a}_{\mathrm{equ}}=\mathrm{a}_{\mathrm{con}} / \sqrt{ } \varepsilon_{\mathrm{r}}
$$
equation:

Depending on ' $\mathbf{a}_{\text {equ }}$ ', where ' $\mathbf{c}$ ' is velocity of light, the cutoff frequency ' $f_{\mathrm{c}}$ ' can be calculated by

$$
f_{c}=\frac{c}{2 a_{\text {equ }} \sqrt{\varepsilon_{r}}}
$$

The diameter ' $\mathbf{d}$ ' and the periodic distance 'p' of vias must be selected such that there are no leakage radiations from them as discussed in [4]. The periodic length must be greater than the diameter of the vias so that the structure is physically realizable.

$$
\begin{aligned}
& p>d \\
& p \leq d
\end{aligned}
$$

Since SIW is a periodic guided wave structure, the electromagnetic bandstop phenomenon will probably appear which should be avoided over the waveguide bandwidth of interest. Therefore the condition shown below should be used to avoid any bandgap effects in the operating bandwidth.

$$
\left(\mathrm{p} / \lambda_{\mathrm{c}}\right)<0.25
$$

A non essential but desirable condition for the manufacturing process is to minimize the number of vias as the production time is proportional to their numbers. So the number of vias should not exceed 20 per wavelength as in the given condition:

$$
\left(\mathrm{p} / \lambda_{\mathrm{c}}\right)>0.05
$$

Center to center distance between vias of both the rows ' $\mathbf{a}$ ' can be calculated using a simple formula given below form [2],[5] and In [2] four other formulas are discussed to determine the value of ' $\mathbf{a}$ '.

$$
a=a_{\text {equ }}+\frac{d^{2}}{0.95 p}
$$
structure.

Using the above design techniques or rules we can design a SIW from its conventional waveguide 


\section{Design Technique For Microstrip TRANSITION}

The SIW fabricated on planar circuits need to be integrated to planar transmission lines like microstrips. For this microstrip and its transition to waveguide must be designed. The width ' $\mathbf{w}$ ' of the microstrip depends upon the height of the dielectric substrate ' $\mathbf{b}$ ', dielectric constant of the substrate ' $\square_{\mathbf{r}}$ ' and impedance of microstrip line ' $\mathbf{Z}_{\mathbf{0}}$ '. The microstrip width by height of dielectric ratio $(\mathbf{w} / \mathbf{b})$ can be determined by equation as given below from [6]:

$$
\frac{\mathbf{w}}{\mathbf{b}}= \begin{cases}\frac{8 e^{A}}{e^{2 A}-2} & \text { for } \mathbf{w} / \mathbf{b}<2 \\ \frac{2}{\pi}\left[B-1-\ln (2 B-1)+\frac{\epsilon_{r}-1}{2 \epsilon_{r}}\left\{\ln (B-1)+0.39-\frac{0.61}{\epsilon_{r}}\right\}\right] & \text { for } \mathbf{w} / \mathbf{b}>2,\end{cases}
$$

Where in equation (8):

$$
\begin{aligned}
& A=\frac{Z_{0}}{60} \sqrt{\frac{\epsilon_{r}+1}{2}}+\frac{\epsilon_{r}-1}{\epsilon_{r}+1}\left(0.23+\frac{0.11}{\epsilon_{r}}\right) \\
& B=\frac{377 \pi}{2 Z_{0} \sqrt{\epsilon_{r}}}
\end{aligned}
$$

In [7] the equations are discussed to determine the effective permittivity ' $\square$ ' and impedance of microstrip line if $(\mathbf{w} / \mathbf{b})$ ratio is known. Fig. 3 show model of microstrip line and microstrip taper.

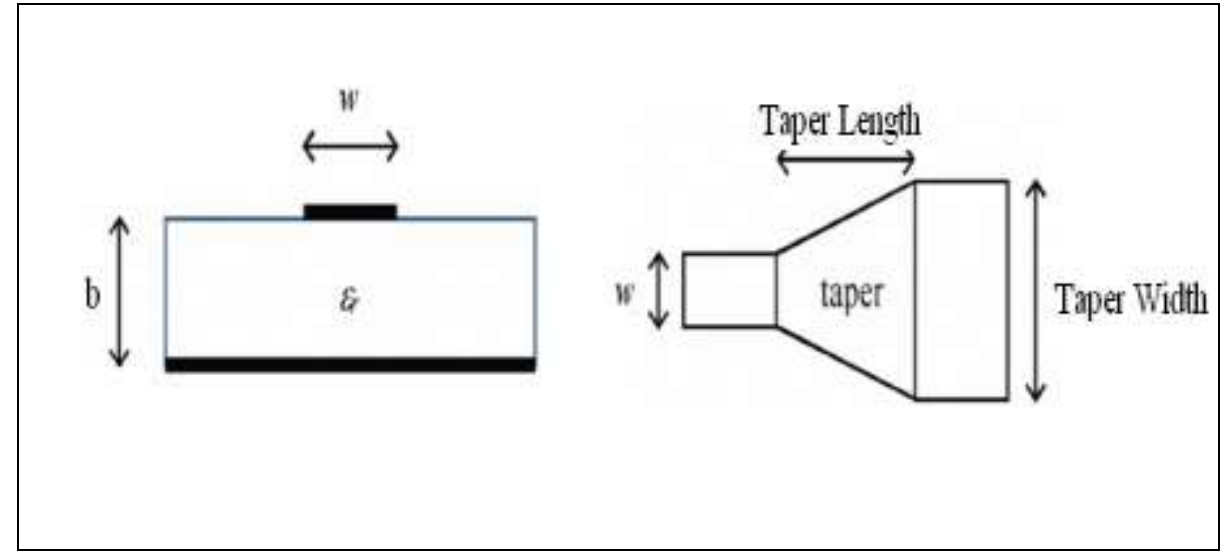

Fig. 3 Model of microstrip line and microstrip taper

Microstrip to waveguide transition is essentially a tapered microstrip line connecting microstrip of width ' $\mathbf{w}$ ' to waveguide of width ' $\mathbf{A}_{\mathbf{g}}$ ' as shown in Fig. 4. We calculate ' $\mathbf{w}$ ' is from equation (8), (9) and (10). Taper width ' $\mathbf{W}_{\mathbf{t}}$ ' and taper length ' $\mathbf{L}_{\mathbf{t}}$ ' are other two essential parameters for microstrip taper design. If ' $\square \square^{\prime}$ ' is the wavelength in microstrip line then ' $\mathbf{W t}$ ' and ' $\mathbf{L t}$ ' can be determined by the relation given below from [8]:

$$
\begin{aligned}
& \mathrm{W}_{\mathrm{t}} / \mathrm{A}_{\mathrm{g}} \approx 0.4 \\
& \left(\lambda_{\mathrm{g}} / 2\right)<\mathrm{L}_{\mathrm{t}}<\lambda_{\mathrm{g}}
\end{aligned}
$$

Where,

$$
\begin{aligned}
& \lambda_{\mathrm{g}}=\lambda_{\mathrm{c}} / \sqrt{ }_{\mathrm{r}_{\mathrm{r}}} \\
& \lambda_{\mathrm{c}}=\mathrm{c} / \mathrm{f}_{\mathrm{c}}
\end{aligned}
$$

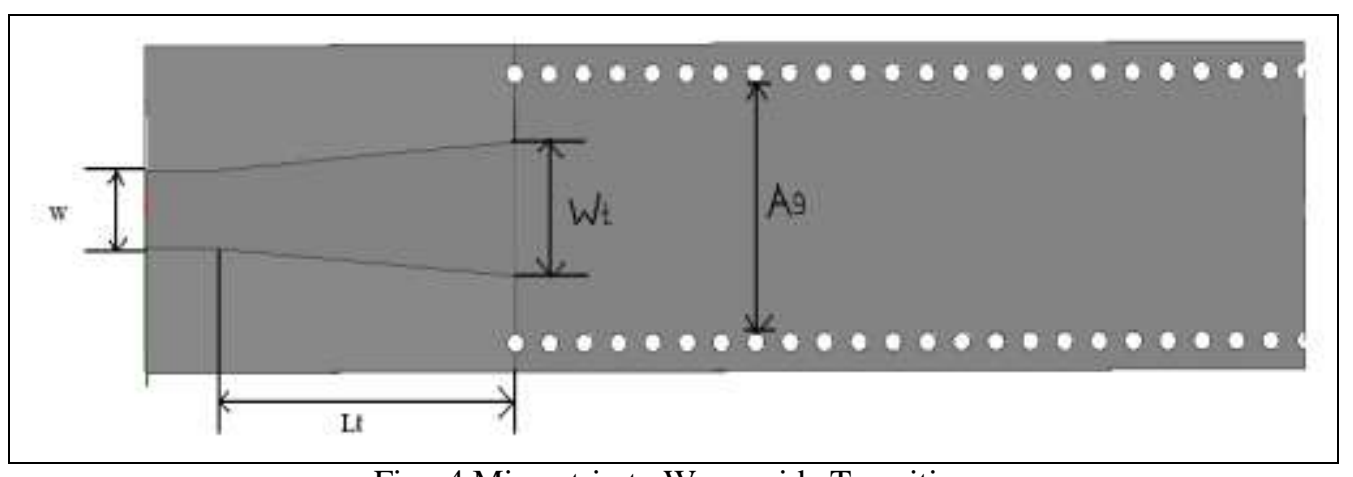

Fig. 4 Microstrip to Waveguide Transition 
Thus using above equations we can design microstrip to waveguide transition. Optimization can be obtained by optimizing ' $\mathbf{W}_{\mathbf{t}}$ ' and ' $\mathbf{L}_{\mathbf{t}}$ ' as discussed in [8].

\section{Example For SIW And Microstrip Taper}

We illustrate a simulation example for SIW and its Microstrip transition. Here the models are designed for $\mathrm{X}$ band $(8 \mathrm{GHz}-12 \mathrm{GHz})$ frequency range. The objective of example is to design models using design techniques, such that reflection parameter is below $-15 \mathrm{~dB}$, good bandwidth is obtained after microstrip to SIW transition and no radiation leakage is present. For designing, conventional waveguide is considered having ' $\square_{\mathbf{c o n}}=\mathbf{1}$ ' and ' $\mathbf{a}_{\text {con' }}$ ' is taken as $\mathbf{1 8 . 7 1} \mathbf{m m}$. Metal thickness is taken as $\mathbf{0 . 0 1} \mathbf{m m}$. Cutoff frequency calculated as 8 $\mathrm{GHz}$ form equation (2), which Lower frequency of $\mathrm{X}$ band and simulation is done for $10 \mathrm{GHz}$ frequency which fall under $\mathrm{X}$ band. Length is in millimeter and frequency is in GHz. Using all the above equations we find the required parameters as shown below in Table 1:

Table 1. Parameters for SIW and Microstrip transition design

\begin{tabular}{|c|c|c|c|c|c|c|c|c|c|c|c|c|c|}
\hline $\mathrm{a}_{\text {equ }}$ & $\mathrm{a}$ & $\mathrm{b}$ & $\mathrm{f}_{\mathrm{c}}$ & $\lambda_{\mathrm{c}}$ & $\lambda_{\mathrm{g}}$ & $\varepsilon_{\mathrm{r}}$ & $\mathrm{p}$ & $\mathrm{d}$ & $\mathrm{A}_{\mathrm{g}}$ & $\mathrm{W}_{\mathrm{t}}$ & $\mathrm{L}_{\mathrm{t}}$ & $\mathrm{w}$ & $\mathrm{Z}_{0}$ \\
\hline 10.8 & 11.83 & 0.508 & 8 & 37.5 & 21.65 & 3 & 2 & 1.4 & 10.43 & 4.17 & 12 & 1.27 & 50 \\
\hline
\end{tabular}

The Simulation results for $S_{11}, S_{12}$ parameters of SIW and its E-Field are shown below in Fig. 5 and Fig. 6 respectively.

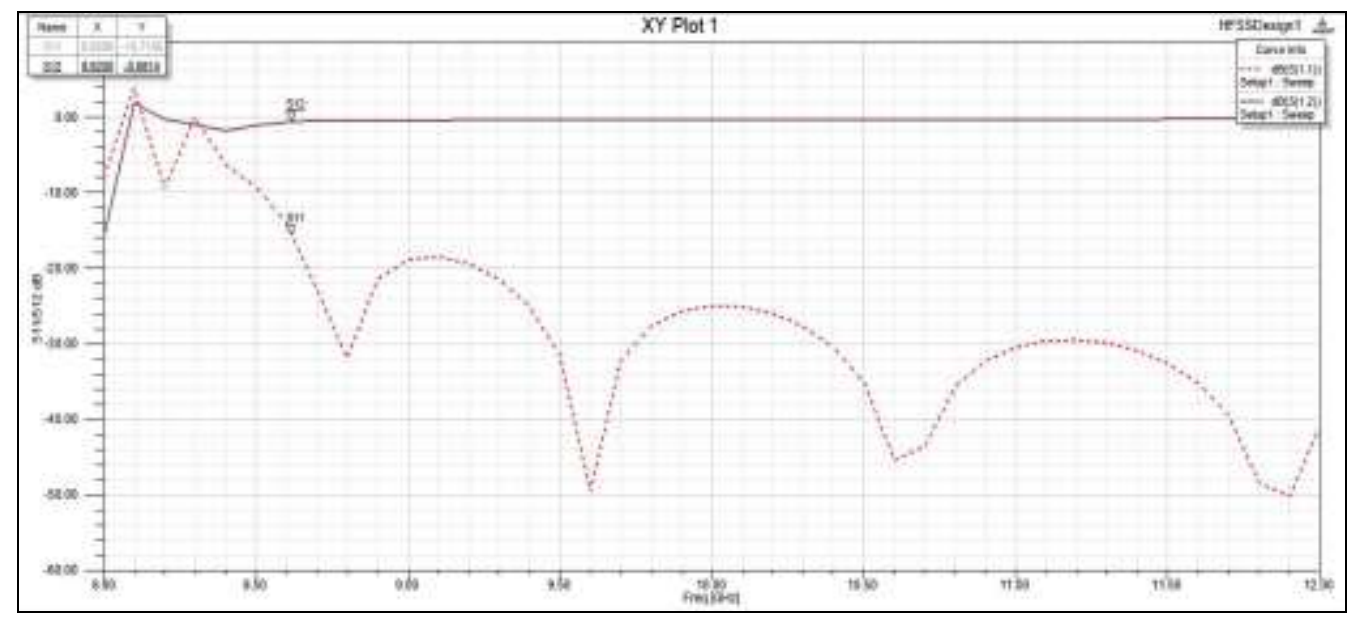

Fig. 5 SIW S-Parameter

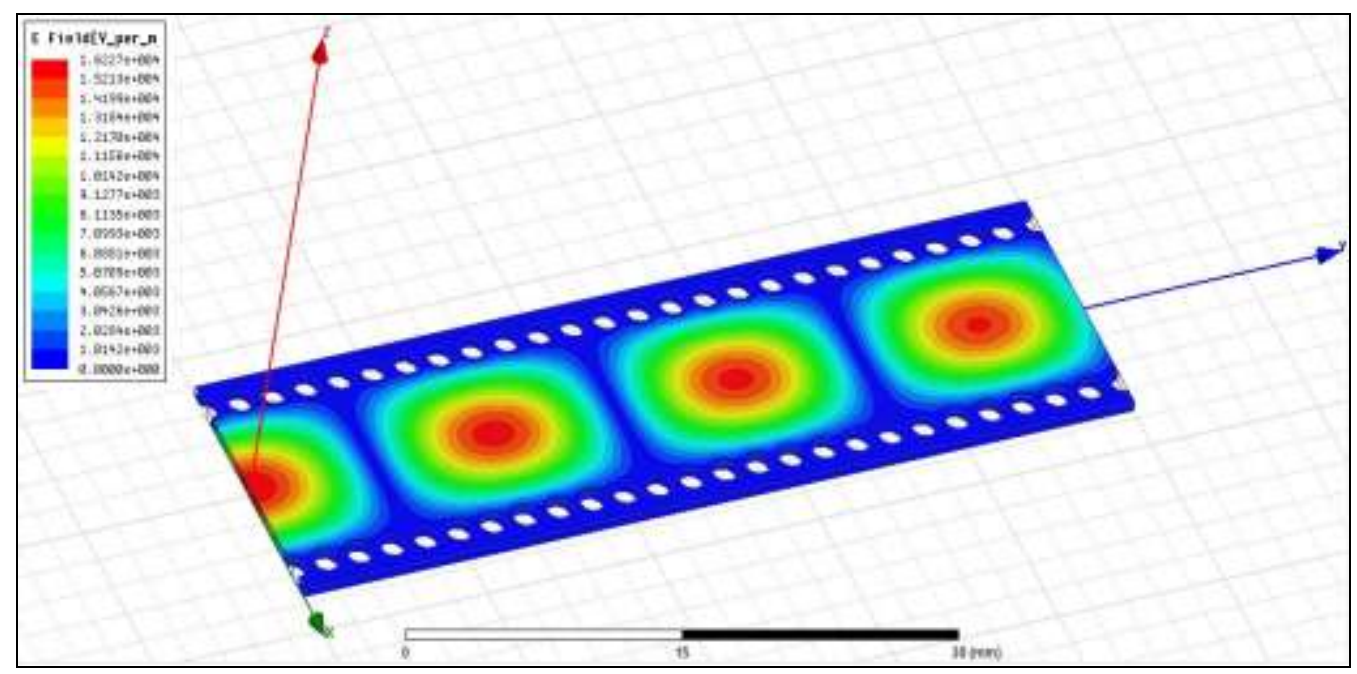

Fig. 6 SIW E-Field Plot at $10 \mathrm{GHz}$

The Simulation results for $S_{11}, S_{12}$ parameters of Microstrip to SIW transition and its E-Field are shown below in Fig. 7 and Fig. 8 respectively. 


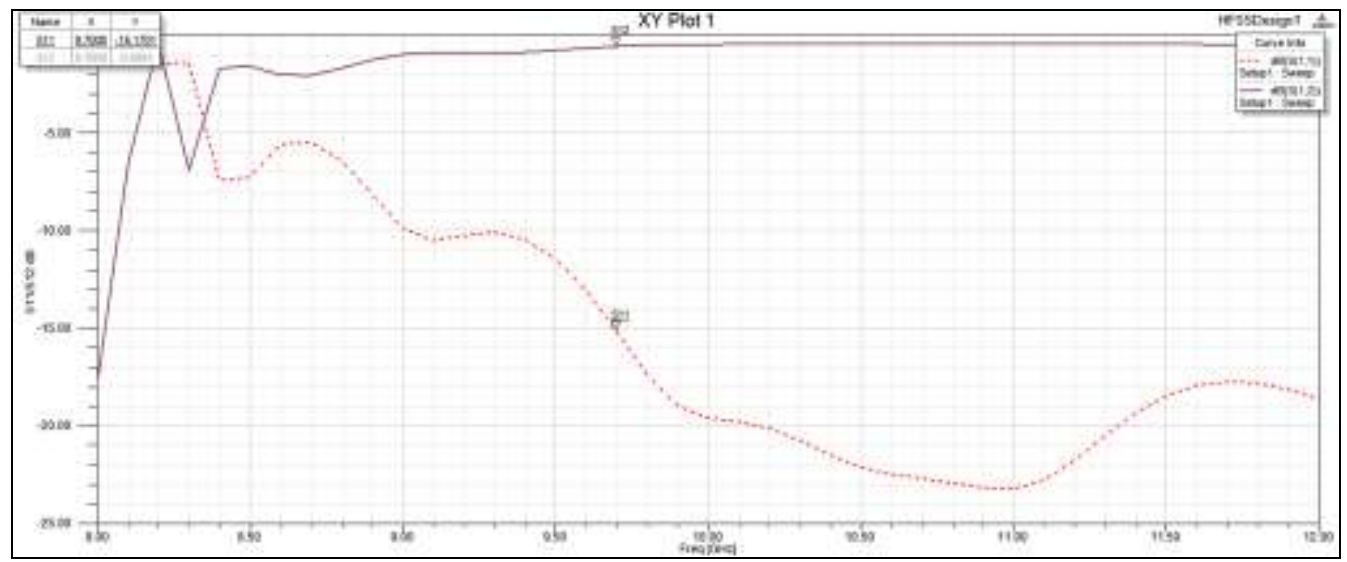

Fig. 7 Microstrip to SIW Transition S-Parameter

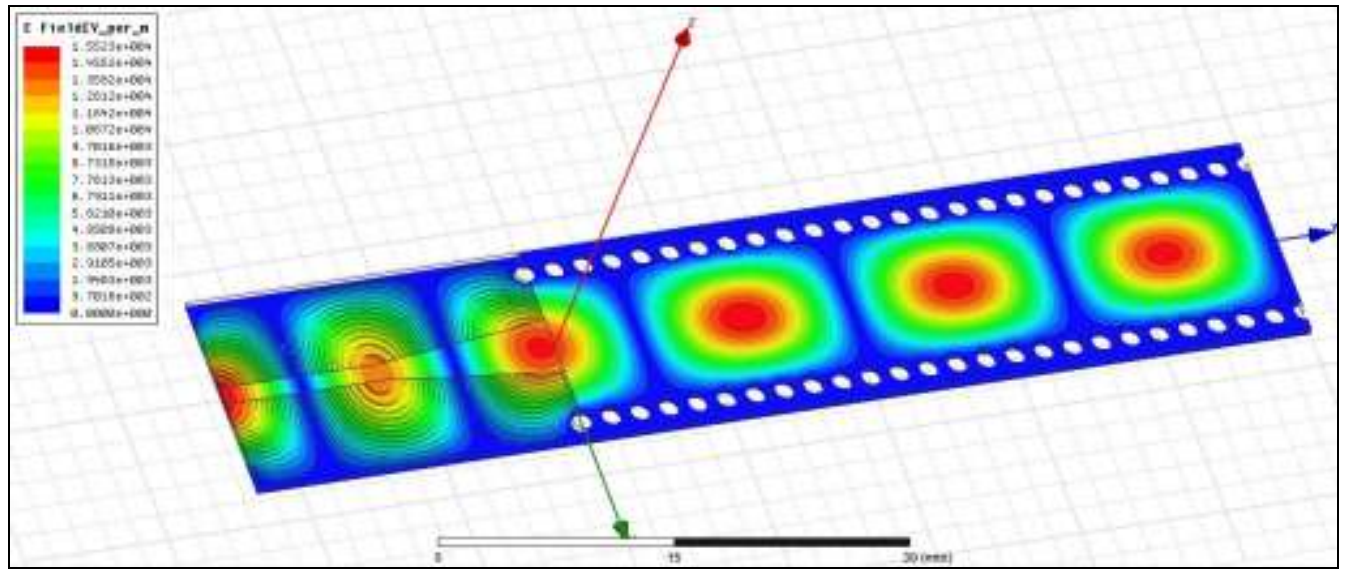

Fig. 8 Microstrip to SIW Transition E-Field at $10 \mathrm{GHz}$

\section{Conclusion}

A Review on Substrate Integrated Waveguide and its Microstrip Interconnect shows that using design techniques, a conventional waveguide can be converted to its SIW equivalent and can be transitioned with microstrip, which can be integrated with planar circuits with ease.

From Simulation results of the discussed example, we get $S_{11}$ parameter of SIW below $-15 \mathrm{~dB}$ from $8.62 \mathrm{GHz}$ onwards and a bandwidth of $3.38 \mathrm{GHz}$, in X Band. After Microstrip to SIW transition, we get $-15 \mathrm{~dB}$ from $9.7 \mathrm{GHz}$ onwards and a bandwidth of $2.3 \mathrm{GHz}$, in X Band. For both models, from simulation results, we can see that the E-Field is contained within SIW and there is no leakage radiation outside the vias of SIW.

SIW can be used in planar circuits with is low radiation loss. System on Substrate (SoS) is a good example where SIW technology can be applied.

\section{References}

[1] Maurizio Bozzi, Luca Perregrini, Ke Wu, and Paolo Arcioni, Current and Future Research Trends in Substrate Integrated Waveguide Technology, RADIOENGINEERING, VOL 18, NO. 2, JUNE 2009.

[2] Farzaneh Taringou and Jens Bornemann, Return-Loss Investigation of Equivalength width of Substrate Integrted Waveguide Circuits, IEEE MTT-S International Microwave Workshop Series on millimeter Wave Integraton Technologies, 2011.

[3] E. Diaz, E. Miralles, H. Esteban, A. Belenguer, V.Boria, C. Bachiller, J.V. Morro and A.L. Borja, Efficient and Accurate Design of Passive Devices in Substrate Integrated Waveguide Technology and their Tapered Transitions from Microstrip Lines, Waves, year 3, 2011.

[4] D. Deslandes and K. Wu, Accurate Modeling, Wave Mechanisms, and Design Considerations of a Substrate Integrated Waveguide, IEEE Trans. Microwave Theory \& Tech., VOL. 54, NO 6, pp. 2516-2526, JUN. 2006

[5] H. Aghayari and N. Komjani, Substrate Integrated Waveguide H-Plane Filter, Proceedings of $39^{\text {th }}$ European Microwave Conference, Rome, Italy, 29 Sep-1 Oct 2009.

[6] David M. Pozar, Microwave Engineering, (John Wiley \& Sons, Inc., Second Edition), section 3.8, pp. 160-163.

[7] Dominic Deslandes, Design Equations for Tapered Microstrip-to-Substrate Integrated Waveguide Transitions, IEEE 2010, IMS 2010, pp 704-707.

[8] Asanee Suntives, High-Speed Data Transmission Using Substrate Integrated Waveguide-Type Interconnects, Degree of Doctor, McGill University, Montreal, Quebec, Canada, Feb 2009. 